\title{
Experimental study of the anti-atherosclerotic effect of demethylzeylasteral
}

\author{
YING HUANG $^{1 *}$, SHAOFENG WANG ${ }^{2 *}$, CHUNYA ZHANG $^{1}$, ZHIQING XU $^{1}$, JINGHUA SHEN $^{1}$, \\ XIAOGANG DU ${ }^{1}$, HUANHUA ZHANG ${ }^{1}$, KANGJIAN ZHANG ${ }^{1}$ and DAIFU ZHANG ${ }^{1}$ \\ ${ }^{1}$ Department of Cardiology, Shanghai University of Medicine and Health Sciences, Pudong New Area People's Hospital; \\ ${ }^{2}$ Department of General Medicine, Huang Lou Community Health Center, Shanghai 201200, P.R. China
}

Received November 4, 2016; Accepted March 17, 2017

DOI: $10.3892 /$ etm.2017.4352

\begin{abstract}
This study aimed to confirm that atherosclerosis (AS) is a systemic immune-mediated chronic inflammatory disease and to investigate the anti-atherosclerotic effect of demethylzeylasteral by testing the immunocompetent cells and inflammatory mediators in the blood and atherosclerotic plaques of the rabbit model of AS. For this purpose, 60 male New Zealand white rabbits were given $150 \mathrm{~g}$ high-fat diet (1\% cholesterol, $5 \%$ lard, and $15 \%$ egg yolk powder) daily for a total of 90 days. On day 61, the rabbits were randomly divided into the saline group $(n=15)$, the rosuvastatin group $(n=15)$, the low-dose demethylzeylasteral group $(n=15)$, and the high-dose demethylzeylasteral group $(\mathrm{n}=15)$. The $\mathrm{CD}^{+} \mathrm{T}$ lymphocytes and the subsets $\mathrm{CD}^{+}, \mathrm{CD}^{+}$, and $\mathrm{CD} 4^{+} / \mathrm{CD}^{+}$, as well as the soluble interleukin-2 receptor (sIL-2R) were measured before and after the treatment. The contents of immunoglobulins $\mathrm{IgG}, \mathrm{IgA}$ and $\mathrm{IgM}$ and the levels of complements $\mathrm{C} 3$ and $\mathrm{C} 4$ were also monitored. In addition, the level of anti-oxidized low-density lipoprotein (ox-LDL) antibody, the inflammatory cytokines tumor necrosis factor- $\alpha$ (TNF- $\alpha)$, IL-6 and metalloproteinase-9 (MMP-9), the blood lipids triglyceride (TG), total cholesterol (TC), LDL cholesterol (LDL-C) and high-density lipoprotein cholesterol (HDL-C) were measured, and the severity of plaque lesions was also evaluated. Our results showed that the saline group, the rosuvastatin group and the low-dose demethylzeylasteral group had significantly lower activated $\mathrm{T}$ lymphocyte parameters $\mathrm{CD}^{+}, \mathrm{CD}^{+}, \mathrm{CD}^{+}$ and $\mathrm{CD}^{+} / \mathrm{CD}^{+}(\mathrm{P}<0.05)$, and significantly higher levels of sIL-2R, immunoglobulins IgG, IgA and IgM, complements
\end{abstract}

Correspondence to: Dr Daifu Zhang or Dr Ying Huang, Department of Cardiology, Shanghai University of Medicine and Health Sciences, Pudong New Area People's Hospital, 490 Chuan Huan South Road, Shanghai 201200, P.R. China

E-mail: zep941@163.com

E-mail: sunllmm2007@126.com

*Contributed equally

Key words: demethylzeylasteral, atherosclerosis, traditional Chinese medicine, immune and inflammatory responses
C3 and C4, anti-ox-LDL antibody, TNF- $\alpha$, IL-6 and MMP-9 $(\mathrm{P}<0.01)$ when compared with the high-dose demethylzeylasteral group. Moreover, TG, TC, LDL-C contents were found significantly lower and their HDL-C contents were significantly higher in high-dose demethylzeylasteral group $(\mathrm{P}<0.01)$ as compared to the other three groups. Furthermore, Sudan staining and haematoxylin and eosin staining of the thoracic aorta showed that, after 30-day treatment, the high-dose demethylzeylasteral group had the smoothest intima and the lightest plaque lesions among the four groups. Based on these results, we concluded that AS is a systemic immune-mediated chronic inflammatory disease and the relatively high dose of demethylzeylasteral used in the treatment of atherosclerotic rabbits could significantly alleviate AS. This implies that demethylzeylasteral may be considered as a suitable drug for anti-immunization therapy.

\section{Introduction}

Atherosclerosis (AS), a chronic progressive disease characterized by fiber and lipid depositions on the artery wall (1), is a major cause of myocardial infarction and stroke. The pathogenesis of AS has not been fully understood yet. Recent studies have shown that immunity and inflammatory responses might be involved in the whole process of AS, and it was even suggested that AS was a kind of autoimmune disease (2-5). Macrophages, $\mathrm{T}$ lymphocytes and many other immune cells have been detected in the AS lesions. In addition, the incidence of AS was significantly higher in patients with autoimmune diseases such as rheumatoid arthritis and systemic lupus erythematosus, and glucocorticoid treatment could alleviate AS (6). The above evidence suggests the existence of similarities between AS and autoimmune diseases.

Coronary heart disease and stroke caused by AS are the main reasons of death in humans. The existing anti-AS drugs primarily reduce low-density lipoprotein cholesterol (LDL-C) (e.g., statins and ezetimibe), and prevent platelet aggregations. However, the effects of these anti-AS drugs are still unsatisfactory. Studies showed that anti-inflammatory and anti-immune drugs such as cyclosporine and methotrexate could reduce or delay the progression of AS (7-10), but whether anti-inflammatory and anti-immune Chinese medicine Tripterygium would reduce or delay AS has not been reported. Therefore, the main 
focus of this study was to explore the anti-atherosclerotic effect of demethylzeylasteral.

\section{Materials and methods}

Animals. For this study, 60 male New Zealand white rabbits (weight, 2.0-2.5 kg) were housed in the SPF level animal facility with one rabbit per cage. Feed and water was given to the animals, and litter and cages were sterilized before use. The indoor temperature was kept at $20-22^{\circ} \mathrm{C}$, and the animals had each day 12-h light and 12-h dark.

Animal feed and demethylzeylasteral intervention. The rabbits were given $150 \mathrm{~g}$ high-fat diet (1\% cholesterol, 5\% lard and $15 \%$ egg yolk powder) daily for 90 days. On day 61, the animals were randomly divided into the saline group, the rosuvastatin group, the low-dose demethylzeylasteral group, and the high-dose demethylzeylasteral group, each consisting of 15 rabbits. The rabbits from each group received saline, $0.5 \mathrm{mg} / \mathrm{kg} /$ day rosuvastatin (National Medicine Permit no. J20090092), $10 \mathrm{mg} / \mathrm{kg} /$ day demethylzeylasteral (provided by the Department of Pharmacy, Zhongshan Hospital of Fudan University, Shanghai, China) and $40 \mathrm{mg} / \mathrm{kg} /$ day demethylzeylasteral, respectively by intragastrical administration. The treatment was continued for 30 days.

Collection of blood samples. Venous blood samples were collected before the high-fat feeding, and before and after the interventions with saline, rosuvastatin and demethylzeylasteral. Ethics approval for animal experiments was from the Animal Ethics Committee of Shanghai University of Medicine and Health Sciences.

Collection of pathology specimen. On day 91, the rabbits were sacrified by air injection after they were given anesthesia by intraperitoneal injection of ketamine $(35 \mathrm{mg} / \mathrm{kg}$ ) and diazepam $(5 \mathrm{mg} / \mathrm{kg})$. Then the abdominal cavities of the animals of each group were opened and the epicardial adipose tissues were peeled from the aortic root to the level of the iliac artery. After that, the aortas were stored in $4 \%$ paraformaldehyde (PBS, $\mathrm{pH}$ 7.4). Then, a $0.5-\mathrm{cm}$ fragment starting from the descending aorta was separated and sliced into $4 \mu \mathrm{m}$ pathology sections for immunohistochemical [hematoxylin and eosin (H\&E)] staining. The rest of the aorta was opened longitudinally and stained with Sudan III.

Measurements of cellular immune indexes. The $\mathrm{CD}^{+}$ $\mathrm{T}$ lymphocytes and the subsets $\mathrm{CD}^{+}, \mathrm{CD}^{+}$, and $\mathrm{CD} 4^{+} / \mathrm{CD} 8^{+}$ were measured by direct immunofluorescence labeling before and after the treatment. An assay (ELISA) was used to measure the soluble interleukin-2 receptor (sIL-2R) with the sIL-2R kit (Meixuan Biotechnology Co., Ltd., Shanghai, China) by following the manufacturer's operating manual.

Determination of humoral immune indexes. The rate nephelometry was used to determine the serum level of $\mathrm{IgG}$, IgA, IgM, C3 and C4.

Measurement of autoimmune index. The anti-oxidized LDL (ox-LDL) antibody was measured by using ELISA with the anti-rabbit ox-LDL antibody ELISA kit (Nanjing SenBeijia Biotechnology Co., Ltd., Nanjing, China) following the instruction of manufacturer's manual.

Determination of inflammatory cytokines. To measure the serum tumor necrosis factor- $\alpha$ (TNF- $\alpha$ ), IL-6, metalloproteinase-9 (MMP-9) contents, radioimmunoassay was used. The relevant kits were purchased from SunBio Biomedical Technology (Beijing, China). The operations were conducted according to the instructions given on the manufacturers' manual.

Determination of blood lipid levels. The contents of blood triglyceride (TG), high-density lipoprotein cholesterol (HDL-C), LDL-C and total cholesterol (TC) were measured on an automatic biochemical analyzer (Shengshida Technology, Wuhan, China) following the instructions' manual of the relevant kits.

Analysis of pathology images. Sudan staining and H\&E staining were used to stain the rabbit thoracic aortas and the degrees of plaque lesions for each group were evaluated.

Statistical analysis. To perform the statistical analysis, the SPSS 20.0 (IBM SPSS, Armonk, NY, USA) was used. Countable data were converted into rates and then compared by using the Chi-square test. The measurement data were expressed as the mean \pm standard deviation. Furthermore, pairwise comparisons were performed by using the t-test, and F test was used to make comparison among groups. Statistical significance was set at $\mathrm{P}<0.05$.

\section{Results}

Comparison of cellular immune indexes. The results presented in Table I shows that the high-dose demethylzeylasteral group had significantly higher activated $\mathrm{T}$ lymphocytes parameters, that is, $\mathrm{CD}^{+}, \mathrm{CD}^{+}, \mathrm{CD}^{+}$, and $\mathrm{CD} 4^{+} / \mathrm{CD}^{+}(\mathrm{P}<0.05)$, but significantly lower sIL-2R level $(\mathrm{P}<0.01)$ as compared to other three groups.

Comparison of humoral immune indexes. The results presented in Table II demonstrate that the saline group, the rosuvastatin group, and the low-dose demethylzeylasteral group showed significantly higher levels of immunoglobulins IgG, IgA and IgM, as well as the levels of complements C3 and C4 than the high-dose demethylzeylasteral group $(\mathrm{P}<0.05)$.

Comparison of autoimmune index. The level of anti-ox-LDL antibody was found significantly higher in the saline group, the rosuvastatin group, and the low-dose demethylzeylasteral group (Fig. 1) than in the high-dose demethylzeylasteral group $(\mathrm{P}<0.05)$.

Comparison of inflammatory cytokines. The results presented in Table III illustrates that the saline group, the rosuvastatin group, and the low-dose demethylzeylasteral group had significantly higher levels of TNF- $\alpha$, IL- 6 and MMP-9 than the high-dose demethylzeylasteral group $(\mathrm{P}<0.01)$. 
Table I. Comparison of cellular immune indexes between groups.

\begin{tabular}{lccccc}
\hline Group & $\mathrm{CD}^{+}, \%$ & $\mathrm{CD}^{+}, \%$ & $\mathrm{CD}^{+}, \%$ & $\mathrm{CD}^{+} / \mathrm{CD} 8^{+}$ & sIL-2R, ng/l \\
\hline Saline & $41.54 \pm 5.32$ & $24.67 \pm 4.85$ & $14.27 \pm 3.18$ & $1.46 \pm 0.52$ & $862.57 \pm 171.28$ \\
Rosuvastatin & $47.73 \pm 7.16$ & $28.12 \pm 5.04$ & $17.05 \pm 4.32$ & $1.61 \pm 0.44$ & $794.23 \pm 153.71$ \\
Low-dose demethylzeylasteral & $51.35 \pm 7.42$ & $32.51 \pm 5.37$ & $19.63 \pm 4.65$ & $1.75 \pm 0.74$ & $726.75 \pm 136.33$ \\
High-dose demethylzeylasteral & $60.05 \pm 8.54$ & $36.85 \pm 5.83$ & $23.04 \pm 5.12$ & $1.93 \pm 0.85$ & $676.72 \pm 125.13$ \\
F & 19.23 & 17.73 & 14.54 & 15.32 & 10.12 \\
P-value & $<0.05$ & $<0.05$ & $<0.05$ & $<0.05$ & $<0.01$
\end{tabular}

sIL-2R, soluble interleukin-2 receptor.

Table II. Comparison of humoral immune indexes between groups.

\begin{tabular}{|c|c|c|c|c|c|}
\hline Group & $\mathrm{IgG}, \mathrm{g} / \mathrm{l}$ & $\operatorname{Ig} \mathrm{A}, \mathrm{g} / \mathrm{l}$ & $\operatorname{IgM}, \mathrm{g} / \mathrm{l}$ & $\mathrm{C} 3, \mathrm{~g} / \mathrm{l}$ & $\mathrm{C} 4, \mathrm{~g} / \mathrm{l}$ \\
\hline Saline & $17.14 \pm 4.27$ & $5.23 \pm 1.16$ & $3.46 \pm 1.25$ & $2.54 \pm 0.92$ & $0.63 \pm 0.18$ \\
\hline Rosuvastatin & $15.73 \pm 4.06$ & $4.85 \pm 0.77$ & $3.07 \pm 1.03$ & $2.35 \pm 0.74$ & $0.52 \pm 0.13$ \\
\hline Low-dose demethylzeylasteral & $12.46 \pm 3.85$ & $3.46 \pm 0.63$ & $2.37 \pm 0.86$ & $1.91 \pm 0.54$ & $0.37 \pm 0.11$ \\
\hline High-dose demethylzeylasteral & $9.66 \pm 3.17$ & $2.28 \pm 0.42$ & $2.14 \pm 0.52$ & $0.83 \pm 0.45$ & $0.24 \pm 0.07$ \\
\hline $\mathrm{F}$ & 5.58 & 9.13 & 9.95 & 4.39 & 5.11 \\
\hline P-value & $<0.05$ & $<0.05$ & $<0.05$ & $<0.05$ & $<0.05$ \\
\hline
\end{tabular}

Table III. Comparison of inflammatory cytokines between groups.

\begin{tabular}{lccc}
\hline Group & TNF- $\alpha, \mathrm{ng} / \mathrm{l}$ & IL-6, pg/ml & MMP-9, ng/ml \\
\hline Saline & $1.45 \pm 0.87$ & $113.45 \pm 15.37$ & $77.37 \pm 13.22$ \\
Rosuvastatin & $1.16 \pm 0.65$ & $97.23 \pm 14.42$ & $66.14 \pm 12.46$ \\
Low-dose demethylzeylasteral & $0.86 \pm 0.51$ & $90.15 \pm 12.36$ & $45.05 \pm 9.67$ \\
High-dose demethylzeylasteral & $0.72 \pm 0.46$ & $76.28 \pm 9.76$ & $34.16 \pm 7.45$ \\
F & 7.67 & 5.47 & 4.79 \\
P-value & $<0.01$ & $<0.01$ & $<0.01$ \\
\hline
\end{tabular}

TNF- $\alpha$, tumor necrosis factor- $\alpha$; IL, interleukin; MMP-9, metalloproteinase-9.

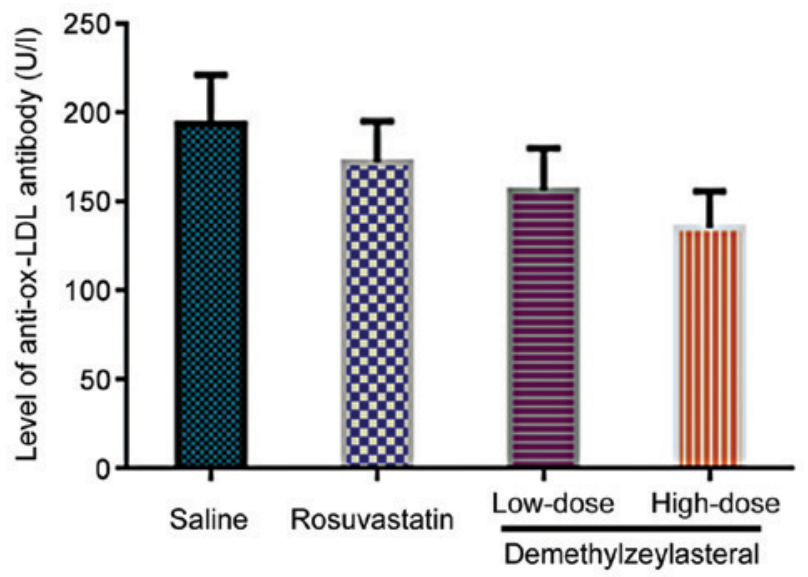

Figure 1. Comparison of autoimmune index between groups. ox-LDL, oxidized low-density lipoprotein.
Comparison of blood lipid levels. As shown in Table IV, the saline group, the rosuvastatin group, and the low-dose demethylzeylasteral group had significantly higher TG, TC, and LDL-C than the high-dose demethylzeylasteral group $(\mathrm{P}<0.01)$, however, their HDL-C content was significantly lower than the high-dose demethylzeylasteral group $(\mathrm{P}<0.01)$.

Evaluation of plaque lesions. The Sudan staining and H\&E staining of the rabbit thoracic aortas after 30-days of treatments showed that the strips and the patches of the orange uplift on the arterial intimal surface in the saline group (Fig. 2), which indicated severe plaque lesions. In case of the rosuvastatin group endometrium, lumps or strips of uplift were found suggesting relatively light plaque lesions (Fig. 3), the low-dose demethylzeylasteral group showed relatively smooth intima 
Table IV. Comparison of blood lipid levels between groups.

\begin{tabular}{lcccc}
\hline Group & TG, mmol/l & TC, mmol/l & HDL-C, mmol/1 & LDL-C, mmol/l \\
\hline Saline & $1.11 \pm 0.11$ & $3.22 \pm 0.32$ & $0.47 \pm 0.06$ & $0.83 \pm 0.12$ \\
Rosuvastatin & $1.04 \pm 0.53$ & $2.85 \pm 0.36$ & $0.51 \pm 0.07$ & $0.74 \pm 0.08$ \\
Low-dose demethylzeylasteral & $1.05 \pm 0.46$ & $2.97 \pm 0.41$ & $0.54 \pm 0.05$ & $0.77 \pm 0.13$ \\
High-dose demethylzeylasteral & $0.93 \pm 0.52$ & $2.63 \pm 0.52$ & $0.63 \pm 0.11$ & $0.62 \pm 0.12$ \\
F & 6.43 & 6.07 & 5.42 & 6.15 \\
P-value & $<0.01$ & $<0.01$ & $<0.01$ & $<0.01$ \\
\hline
\end{tabular}

TG, triglyceride; TC, total cholesterol; HDL-C, high-density lipoprotein cholesterol; LDL-C, low-density lipoprotein cholesterol.
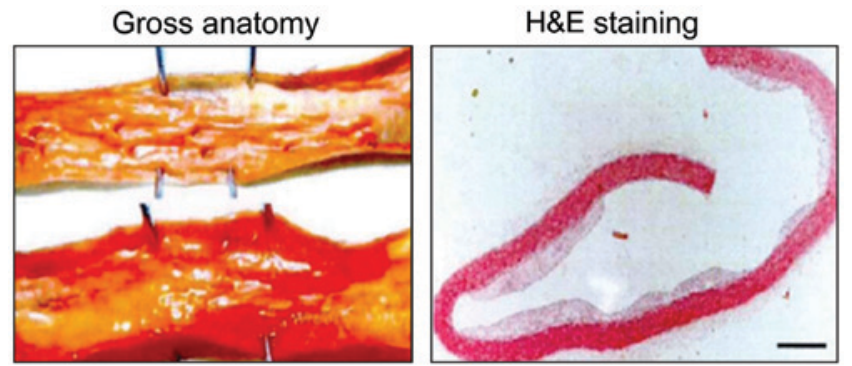

Figure 2. Sudan staining and haematoxylin and eosin (H\&E) staining of rabbit thoracic aorta in the saline group (scale, $400 \mu \mathrm{m}$ ).
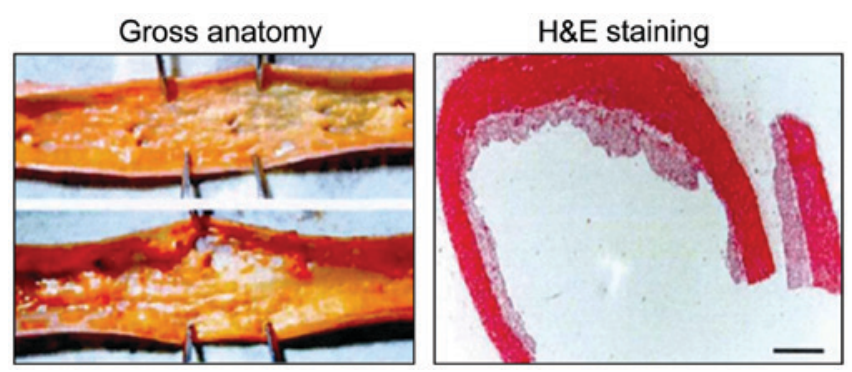

Figure 3. Sudan staining and haematoxylin and eosin (H\&E) staining of rabbit thoracic aorta in the rosuvastatin group (scale, $400 \mu \mathrm{m}$ ).

with spotted uplift suggesting light plaque lesions (Fig. 4), and the smoothest intima and the lightest plaque lesions were found in the high-dose demethylzeylasteral group (Fig. 5).

\section{Discussion}

The immune system is activated in the process of AS formation, and the individual members of the immune system play an important role in AS (11). Studies have shown the existence of a large number of immune cells, for example, macrophages and lymphocytes, at the AS lesion sites (11-13). The macrophages can generate oxygen radicals and proteases, and can intake non-specific immune responses mediated by lipoprotein through scavenger receptors. In addition, macrophages can present exogenous antigens to $\mathrm{T}$ lymphocytes to initiate specific immune responses. Clinical studies discovered that lymphocytes accounted for 10-20\% of nucleated cells in atherosclerotic plaques (13). Futhermore, lymphocytes are divided into T-lymphocytes, B-lymphocytes and NK cells,
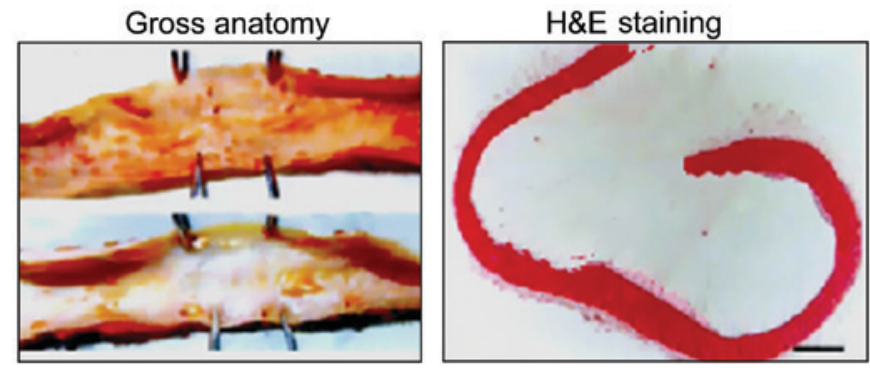

Figure 4. Sudan staining and haematoxylin and eosin (H\&E) staining of rabbit thoracic aorta in the low-dose demethylzeylasteral group (scale, $400 \mu \mathrm{m}$ ).
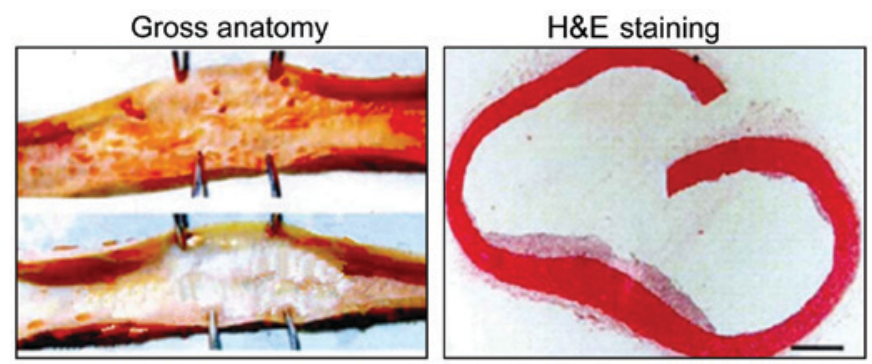

Figure 5. Sudan staining and haematoxylin and eosin (H\&E) staining of rabbit thoracic aorta in the high-dose demethylzeylasteral group (scale, $400 \mu \mathrm{m}$ ).

where T-lymphocytes $\left(\mathrm{CD}^{+}\right)$can further be divided into two main groups, namely, T help/inducer cells $\left(\mathrm{CD}^{+}\right)$and $\mathrm{T}$ suppressor/cytotoxic cells $\left(\mathrm{CD}^{+}\right)$. It is found that during the development of atherosclerotic plaques, $\mathrm{T}$ lymphocytes enhance the local immune responses, promote phagocytosis of macrophages and secret cytokines and growth factors, and at the same time, it also promotes the proliferation, migration, and phagocytosis of medial vascular smooth muscle cells (14). Activated $\mathrm{CD}^{+} \mathrm{T}$ lymphocytes produce a large amount of interferon- $\gamma$ (INF- $\gamma$ ), TNF- $\alpha$ and other pro-inflammatory cells, and thus affect the metabolisms of the extracellular matrix and the functions of endothelial cells. The activation of $\mathrm{CD} 8^{+} \mathrm{T}$ cells actually kills adjacent cells by physical contacts. In other words, all the medium produced in the process aggregates CD $8^{+}$cells to kill smooth muscle cells and macrophages, which can promote the process of AS, and cause plaque ruptures and other complications $(15,16)$. The activation of NK cells produce proinflammatory cytokines and promote AS. Humoral immunity was also involved in the formation 
of AS. Normal artery walls had no immunoglobulins, while deposited immunoglobulins were found in AS lesions, which suggest that immunoglobulins were involved in the occurrence of AS. Complements are important components of the innate immunity. Products generated during complement activation are common effector molecules shared by specific immunity and nonspecific immunity, and play important roles in the body's resistance to infection, immune regulation, and immune surveillance, however, under certain circumstances, they can also cause tissue damage. It is found (17-20) that lesions of AS had not only complements depositions but also activated compliments resulting in damaged tissues.

Anti-inflammatory and anti-immune drugs include nonsteroidal anti-inflammatory drugs, cyclooxygenase-2 inhibitors, cyclosporine, methotrexate and traditional Chinese medicines, Tripterygium is considered the strongest immunosuppressive Chinese medicine, which have fewer side effects than western medicines (21). The plant Tripterygium belongs to the Celastraceae family and has been used as a pesticide for nearly two thousand years. Recently, it is found that Tripterygium has anti-inflammatory and immune suppression effects, and it has gradually been used for the treatment of autoimmune diseases, including rheumatoid arthritis, ankylosing spondylitis and systemic lupus erythematosus (22-25). Over 150 kinds of ingredients, mainly alkaloids, diterpenoids, triterpenoids, sesquiterpene and carbohydrates have been isolated from Tripterygium plants, including demethylzeylasteral (T96), a triterpene monomer, which is isolated from the root bark of Tripterygium by the Department of Pharmacy, Zhongshan Hospital of Fudan University. Compared with other currently known monomeric compounds isolated from Tripterygium such as triptolide, celastrol and Tripterygium glycosides, demethylzeylasteral demonstrated the strongest anti-immune effects and lowest toxicity. Note that the T cells are the central part of the entire immune response. The immune intervention targeted at $\mathrm{T}$ cells can effectively control the body's immune damage. Tripterygium could regulate $\mathrm{T}$ cells from many aspects. Tripterygium and its extracts could inhibit $\mathrm{T}$ cell proliferation. For instance, $\mathrm{Xu}$ et al (26) used mitogen ConA to stimulate splenocytes. The addition of T96 was found to be able to inhibit the transformation of spleen cells and the functions of $\mathrm{T}$ and $\mathrm{B}$ lymphocytes, and the increase in dose of T96 can enhance the degree of inhibition. Pan et al (27) studied PG490-88 (a semi-synthetic derivative of triptolide) on graft-versus-host disease (GVHD) and the in vitro experiments found that when $\mathrm{V} \beta 3^{+}$cells were in the logarithmic amplification phase, PG490-88 could inhibit the expression of spleen $\mathrm{CD}^{+}$ $\mathrm{V} \beta 3^{+}$and $\mathrm{CD}^{+} \mathrm{V} \beta 3^{+} \mathrm{T}$ cells and may thus inhibit the expansion of alloreactive $\mathrm{T}$ cells, thereby preventing the occurrence of GVHD. Tripterygium can also inhibit $\mathrm{T}$ cell proliferation induced by antigen, superantigen and mitogen by exerting its pharmacological effects by regulating $\mathrm{CD}^{+}, \mathrm{CD}^{+}$and $\mathrm{CD} 8^{+}$ cells, and the $\mathrm{CD}^{+} / \mathrm{CD}^{+}$ratio. Moreover, Tripterygium can inhibit the proliferation of B cells and the production of immunoglobulins, as well as the production of antibodies. It was also proposed (28) that Tripterygium could inhibit the expression of CD40 mediated by ionomycin and may thus inhibit the growth and differentiation of B cells and the class switching of immunoglobulins, and suppress the humoral immunity. Note that Tripterygium and its extracts can regulate a variety of cytokines in the body. Triptolide inhibits inflammation by inhibiting the generation of TNF- $\alpha$ and therefore the production of IL-6. It can also act on the transcription level of IL-2 and its receptor gene, inhibiting the expression of both of them. The transcription inhibition suppresses the immune responses mediated by IL-2 cells and thereby limit the clonal expansion of activated T cells. The Th1 cells mainly secrete INF- $\gamma$ and other pro-inflammatory cytokines, while the Th2 cells secrete IL-4 and other anti-inflammatory factors. It is found that the inhibition by Tripterygium is stronger on Th2 than on Th1, which helps to maintain the ratio of Th1/Th2 and balance the pro-inflammatory anti-inflammatory functions. In addition, triptolide TZ93 significantly inhibits the activity of NK cells in the peripheral blood mononuclear cells after PHA-stimulation. It may inhibit lymphocyte activation like other substances, which influence the NK cell activity by inhibiting the secretion of IL-2 and the expression of IL-2 receptors $(29,30)$. In summary, Tripterygium and its extracts can regulate the body's immune functions by affecting the proliferation and activation of $\mathrm{T}$ and $\mathrm{B}$ cells, adjusting the ratio of regulatory $\mathrm{T}$ cell subsets, modulating the production of immunoglobulins and NK cells, and influencing the secretion of various cytokines.

Our study showed that the saline group, the rosuvastatin group and the low-dose demethylzeylasteral group had significantly lower activated $\mathrm{T}$ lymphocyte parameters $\mathrm{CD}^{+}, \mathrm{CD}^{+}$, $\mathrm{CD}^{+}$and $\mathrm{CD}^{+} / \mathrm{CD}^{+}(\mathrm{P}<0.05)$, and significantly higher levels of sIL-2R, immunoglobulins IgG, IgA and IgM, complements C3 and C4, anti-ox-LDL antibody, TNF- $\alpha$, IL-6 and MMP-9 $(\mathrm{P}<0.01)$ when compared with the high-dose demethylzeylasteral group. Moreover, TG, TC, LDL-C contents were found significantly lower and their HDL-C contents were significantly higher in high-dose demethylzeylasteral group $(\mathrm{P}<0.01)$ than the other three groups. Furthermore, Sudan staining and $\mathrm{H} \& \mathrm{E}$ staining of rabbit thoracic aortas after 30-day treatments showed that the high-dose demethylzeylasteral group had the smoothest intima, indicating the lightest plaque lesions among the four groups. These results suggest that AS is a systemic immune-mediated chronic inflammatory disease and the high dose of demethylzeylasteral is more effective in alleviating AS than low-dose demethylzeylasteral and rosuvastatin, and thus it may be considered as a suitable choice for anti-immunization therapy.

\section{Acknowledgements}

This study was supported by the Key Project of Natural Science from Shanghai Health Medical School (no. HMSF-16-21-029).

\section{References}

1. Danoff A, Kendall MA, Currier JS, Kelesidis T, Schmidt AM and Aberg JA: Soluble levels of receptor for advanced glycation endproducts (rage) and progression of AS in individuals infected with human immunodeficiency virus: Actg nwcs 332. Inflammation 39: 1354-1362, 2016.

2. Libby P, Lichtman AH and Hansson GK: Immune effector mechanisms implicated in atherosclerosis: from mice to humans. Immunity 38: 1092-1104, 2013.

3. Hammad SM, Twal WO, Barth JL, Smith KJ, Saad AF, Virella G, Argraves WS and Lopes-Virella MF: Oxidized LDL immune complexes and oxidized LDL differentially affect the expression of genes involved with inflammation and survival in human U937 monocytic cells. Atherosclerosis 202: 394-404, 2009. 
4. Bot PT, Hoefer IE, Piek JJ and Pasterkamp G: Hyaluronic acid: Targeting immune modulatory components of the extracellular matrix in atherosclerosis. Curr Med Chem 15: 786-791, 2008.

5. Vázquez-Agell M, Sacanella E, Tobias E, Monagas M, Antúnez E, Zamora-Ros R, Andrés-Lacueva C, Lamuela-Raventós RM, Fernández-Solá J, Nicolás JM, et al: Inflammatory markers of atherosclerosis are decreased after moderate consumption of cava (sparkling wine) in men with low cardiovascular risk. J Nutr 137: 2279-2284, 2007.

6. Roman MJ, Shanker BA, Davis A, Lockshin MD, Sammaritano L, Simantov R, Crow MK, Schwartz JE, Paget SA, Devereux RB and Salmon JE: Prevalence and correlates of accelerated atherosclerosis in systemic lupus erythematosus. N Engl J Med 349: 2399-2406, 2003.

7. Faco MM, Leone C, Campos LM, Febrônio MV, Marques HH and Silva CA: Risk factors associated with the death of patients hospitalized for juvenile systemic lupus erythematosus. Braz J Med Biol Res 40: 993-1002, 2007.

8. Salisbury D and Bronas U: Inflammation and immune system contribution to the etiology of atherosclerosis: Mechanisms and methods of assessment. Nurs Res 63: 375-385, 2014.

9. Tellides $G$ and Pober JS: Inflammatory and immune responses in the arterial media. Circ Res 116: 312-322, 2015.

10. Lin G, Chen S, Lei L, You X, Huang M, Luo L, Li Y, Zhao X and Yan F: Effects of intravenous injection of Porphyromonas gingivalis on rabbit inflammatory immune response and Atherosclerosis. Mediators Inflamm 2015: 364391, 2015.

11. Kurts C, Panzer U, Anders HJ and Rees AJ: The immune system and kidney disease: Basic concepts and clinical implications. Nat Rev Immunol 13: 738-753, 2013.

12. Liuzzo G, Giubilato G and Pinnelli M: T cells and cytokines in atherogenesis. Lupus 14: 732-735, 2005.

13. Pyka-Fosciak G, Jawien J, Gajda M, Jasek E and Litwin JA: Effect of nebivolol treatment on atherosclerotic plaque components in apoE-knockout mice. J Physiol Pharmacol 64: 745-750, 2013

14. Kyaw T, Winship A, Tay C, Kanellakis P, Hosseini H, Cao A, Li P, Tipping P, Bobik A and Toh BH: Cytotoxic and proinflammatory $\mathrm{CD} 8^{+} \mathrm{T}$ lymphocytes promote development of vulnerable atherosclerotic plaques in apoE-deficient mice. Circulation 127: $1028-1039,2013$

15. Kolumam GA, Thomas S, Thompson LJ, Sprent J and Murali-Krishna K: Type I interferons act directly on CD8 T cells to allow clonal expansion and memory formation in response to viral infection. J Exp Med 202: 637-650, 2011.

16. Wang L, Li D, Yang K, Hu Y and Zeng Q: Toll-like receptor-4 and mitogen-activated protein kinase signal system are involved in activation of dendritic cells in patients with acute coronary syndrome. Immunology 125: 122-130, 2008.

17. Yamashita T, Kasahara K, Sasaki N and Hirata KI: Prevention of atherosclerosis via intervenions for the gut microbiota and modulation of the intestinal immune system. J Intestinal Microbiology 28: 1-5, 2014

18. Zhou L, Song H, Xu W, Xu J, Jiang J, Gong Z, Liu Y, Yan W and Wang L: Immune function of peripheral $T$ cells in patients with venous thromboembolism or coronary artery atherosclerosis. Rev Port Cardiol 33: 339-344, 2014.
19. Zarzycka B, Nicolaes GA and Lutgens E: Targeting the adaptive immune system: New strategies in the treatment of atherosclerosis. Expert Rev Clin Pharmacol 8: 297-313, 2015.

20. Wang Q, Liu D, Song P and Zou MH: Tryptophan-kynurenine pathway is dysregulated in inflammation, and immune activation. Front Biosci (Landmark Ed) 20: 1116-1143, 2015.

21. Tao X, Younger J, Fan FZ, Wang B and Lipsky PE: Benefit of an extract of Tripterygium Wilfordii Hook F in patients with rheumatoid arthritis: a double-blind, placebo-controlled study. Arthritis Rheum 46: 1735-1743, 2002.

22. Lv QW, Zhang W, Shi Q, Zheng WJ, Li X, Chen H, Wu QJ, Jiang WL, Li HB, Gong L, et al: Comparison of Tripterygium wilfordii Hook $\mathrm{F}$ with methotrexate in the treatment of active rheumatoid arthritis (TRIFRA): A randomised, controlled clinical trial. Ann Rheum Dis 74: 1078-1086, 2015.

23. Chen F, Li T, Li S, Hou K, Liu Z, Li L, Cui G, Zu Y and Yang L: Preparation and characterization of Tripterygium wilfordii multi-glycoside nanoparticle using supercritical anti-solvent process. Int J Mol Sci 15: 2695-2711, 2014.

24. He WZ, Yin ZH, Gao JH, Ye ZZ, Xie Y, Kong WH and Chen YS: Etanercept combined with Tripterygium wilfordii polyglycoside for treatment of rheumatoid arthritis in the elderly: A clinical study. Zhongguo Zhong Xi Yi Jie He Za Zhi 34: 267-271, 2014 (In Chinese).

25. Wan YG, Che XY, Sun W, Huang YR, Meng XJ, Chen HL, Shi XM, Tu Y, Wu W and Liu YL: Low-dose of multi-glycoside of Tripterygium wilfordii Hook. f., a natural regulator of TGF- $\beta 1 /$ Smad signaling activity improves adriamycin-induced glomerulosclerosis in vivo. J Ethnopharmacol 151: 1079-1089, 2014.

26. Xu W, Lin Z, Yang C, Zhang Y, Wang G, Xu X, Lv Q, Ren Y and Dong Y: Immunosuppressive effects of demethylzeylasteral in a rat kidney transplantation model. Int Immunopharmacol 9: 996-1001, 2009.

27. Pan F, Fisniku O, Wynn C, Erickson LM, Crews G, Jang MS, Sudo Y, Tamura K, Kobayashi M, Benediktsson H, et al: PG490-88, a new immunosuppressant, effectively prevents acute and chronic rejection in rat renal allografts. Transplant Proc 37: 134-136, 2005

28. Wang W, Bai L, Qiao H, Lu Y, Yang L, Zhang J, Lin R, Ren F, Zhang $\mathrm{J}$ and $\mathrm{Ji} \mathrm{M}$ : The protective effect of fenofibrate against TNF- $\alpha$-induced CD40 expression through SIRT1-mediated deacetylation of NF- $\mathrm{NB}$ in endothelial cells. Inflammation 37: $177-185,2014$.

29. Sun L, Zhang S, Jiang Z, Huang X, Wang T, Huang X, Li H and Zhang L: Triptolide inhibits COX-2 expression by regulating mRNA stability in TNF- $\alpha$-treated A549 cells. Biochem Biophys Res Commun 416: 99-105, 2011.

30. Park B, Sung B, Yadav VR, Chaturvedi MM and Aggarwal BB Triptolide, histone acetyltransferase inhibitor, suppresses growth and chemosensitizes leukemic cells through inhibition of gene expression regulated by TNF-TNFR1-TRADD-TRAF2-NIK-TA K1-IKK pathway. Biochem Pharmacol 82: 1134-1144, 2011. 